\title{
Symmetries in noncommutative field theories: Hopf versus Lie *
}

\author{
D. V. Vassilevich ${ }^{\dagger}$ \\ CMCC - Universidade Federal do ABC, Santo André \\ CEP 09090-400, S.P., Brazil
}

\begin{abstract}
I discuss motivations for introducing Hopf algebra symmetries in noncommutative field theories and briefly describe twisting of main symmetry transformations. New results include an extended list of twisted gauge invariants (which may help to overcome the problem of inconsistency of equations of motion) and a gauge-covariant twist operator (leading to a gauge-covariant star product).

MSC: $81 \mathrm{~T} 75,70 \mathrm{~S} 10$
\end{abstract}

\section{Introduction}

The last decade has witnessed an ever growing interest to noncommutative (NC) field theories [19] motivated by a number of physical applications. In such theories, the coordinates on the space-time manifold $\mathcal{M}$ do not commute, meaning that the algebra of functions on $\mathcal{M}$ is deformed to an associative but not commutative algebra. This reminds us of a very interesting development in mathematics, namely of the discovery of $\mathrm{NC}$ geometry [18].

Symmetry is a guiding principle for constructing field theories. General properties of symmetry transformations are discussed in sec. 1.1. Moyaltype noncommutativity is introduced in sec. 1.2. These two sections are written for non-experts and can be omitted by a more experienced reader.

In sec. 2 we give an overview of several attempts to deform the symmetries together with the algebra of functions without deforming the Leibniz rule (which is the way in which symmetry generators act on products).

\footnotetext{
* Based on a talk given at the Workshop on Quantum Field Theory and Representation Theory, São Paulo, August 21-24, 2007.

$\dagger$ On leave from V. A. Fock Institute of Physics, St. Petersburg University, Russia. E.mail: dmitry (at)dfn.if .usp.br.
} 
We find that neither of this attempts was fully successful. Either only a part of the symmetries can be preserved, or the price to pay is the absence of a closed form of the action.

In sec. 3 we introduce twisted symmetries which eventually lead to a Hopf algebra structure of the symmetry transformations. We then discuss features and problems of this approach focussing on twisted gauge symmetries. We outline solutions of some of the problems (which are new results first reported in this article).

1.1. Symmetries in field theory. To define a classical field theory one needs a smooth manifold $\mathcal{M}$, called the space-time. In this paper we shall consider a real plane $\mathbb{R}^{n}$ and a real torus $\mathbb{T}^{n}$ exclusively. Fields are sufficiently smooth sections of a vector bundle over $\mathcal{M}$. The dynamics of classical field theory is governed by an action $S$, which is a functional of fields. All quantities entering $S$ are subdivided into dynamical variables and parameters. The difference between them is that one has to vary the action with respect to the dynamical variables in order to obtain equations of motion, while the parameters are kept constant. To distinguish between dynamical and non-dynamical variables one either uses some outside knowledge (for example, one knows that electric charge of the proton is always the same, so that it does not make sense to vary it in the action), or checks whether resulting equations describe a meaningful dynamics.

Let us consider a simple example. Let $\mathcal{M}$ is a two-dimensional Minkowski space. Consider a scalar field $\phi$ (a section of trivial line bundle) with the action

$$
S=\int d x d t\left(\left(\partial_{t} \phi\right)^{2}-\left(\partial_{x} \phi\right)^{2}-m^{2} \phi^{2}\right)
$$

which describes free propagation of a massive spinless particle. Vanishing of the variation of $S$ with respect to $\phi$ yields a wave equation $0=\delta S / \delta \phi=$ $\left(-\partial_{t}^{2}+\partial_{x}^{2}-m^{2}\right) \phi$. Variation of $S$ with respect to $m^{2}$ produces the condition $\int d x d t \phi^{2}=0$ which has only a trivial solution $\phi \equiv 0$. This confirms that mass of the particle must not be varied.

Symmetries are very important in physics. Symmetry is a transformation of the dynamical variables which leaves the action invariant. Parameters in the action are not transformed.

The most important global symmetry is the Poincare symmetry. The invariance with respect to global translations is assured by the translational invariance of the space-time integration. The action is invariant with respect to the rotations and Lorentz boosts if all vector indices are contracted in pairs with the help of an invariant bi-linear form. By making translations 
local (i.e. position-dependent) one obtains the diffemorphism transformations. Any Poincare-invariant action can be made also diffeomorphism invariant by introducing a (pseudo-) Riemannian metric to contract indices, and corresponding connection and volume element.

Fundamental interactions of the Standard Model of elementary particles correspond to gauge symmetries with the gauge group $S U(3) \times S U(2) \times$ $U(1)$. All matter fields belong to some unitary representations of this gauge group. Under infinitesimal gauge transformations they transform as $\phi(x) \rightarrow \phi+\delta_{\alpha} \phi, \delta_{\alpha} \phi=\alpha(x) \phi(x)$, where $\alpha(x)=\alpha_{a}(x) T^{a}$ with $T^{a}$ being the generators of corresponding Lie algebra. Gauge fields $A_{\mu}$ then correspond to the connections. They have values in the Lie algebra and transform according to the rule $\delta_{\alpha} A_{\mu}=-\partial_{\mu} \alpha+\left[\alpha, A_{\mu}\right]$. It is easy to check that that $\operatorname{tr}\left(F_{\mu \nu} F^{\mu \nu}\right)$ with $F_{\mu \nu}=\partial_{\mu} A_{\nu}-\partial_{\nu} A_{\mu}+\left[A_{\mu}, A_{\nu}\right]$ is gauge invariant.

1.2. Noncommutative geometry and field theory. To describe an NC deformation of a given manifold $\mathcal{M}$ one takes the algebra $\mathcal{A}$ of smooth functions on $\mathcal{M}$ and deforms is to an algebra $\mathcal{A}_{\theta}$ which is usually assumed to be associative but not commutative. In the sense of Gelfand and Naimark this algebra defines an NC manifold. Practically, one takes the point-wise product $\mu: \mathcal{A} \otimes \mathcal{A} \rightarrow \mathcal{A}, \mu\left(f_{1} \otimes f_{2}\right)(x)=f_{1}(x) \cdot f_{2}(x)$ and replaces it with a deformed product $\mu_{\star}$.

Let us now construct an NC version ${ }^{1}$ of $\mathbb{T}^{n}$ and $\mathbb{R}^{n}$ with the Moyal product (also called the Weyl-Moyal of the Groenewald-Moyal product). Consider a twist operator

$$
\mathcal{F}=\exp \mathcal{P}, \quad \mathcal{P}=-\frac{i}{2} \theta^{\mu \nu} \partial_{\mu} \otimes \partial_{\nu}
$$

(which is indeed a twist, i.e. it satisfies the twist condition, see eq. (9) below). $\theta^{\mu \nu}$ is a skew-symmetric matrix (called an NC parameter). The Moyal product $\mu_{\star}$ is then obtained by twisting the point-wise product:

$$
\phi_{1} \star \phi_{2} \equiv \mu_{\star}\left(\phi_{1} \otimes \phi_{2}\right) \equiv \mu \circ \mathcal{F}^{-1}\left(\phi_{1} \otimes \phi_{2}\right)
$$

The algebra of smooth functions on $\mathbb{R}^{n}$ or $\mathbb{T}^{n}$ equipped with this star product is associative but not commutative.

To construct an NC counterpart of a usual (commutative) field theory one takes an action and replaces all point-wise products by the starproducts. Of course, this prescription fails to give a unique result since the expressions like $\phi_{1} \star \phi_{2}-\phi_{2} \star \phi_{1}$ vanish in the commutative limit. It is natural to require that at least the number of global and local symmetries is preserved by the deformation.

\footnotetext{
${ }^{1} \mathrm{~A}$ more refined construction of the $\mathrm{NC}$ torus can be found in Ref. [17].
} 


\section{Troubles with Lie algebra symmetries}

The problem with symmetries in NC models is that the matrix $\theta^{\mu \nu}$ is a parameter rather than a dynamical variable. Therefore, $\theta^{\mu \nu}$ must not be transformed. However, $\theta^{\mu \nu}$ enters the twist operator $(2)$ is if it were a tensor. Precisely this inconsistency makes it impossible to preserve usual Poincare and diffeomorphism invariances in NC theories.

Consider gauge transformations in the commutative case. Let us take two infinitesimal gauge transformations with the parameters $\alpha(x)=\alpha_{a}(x) T^{a}$, $\beta(x)=\beta_{a}(x) T^{a}$. Their commutator

$$
[\alpha(x), \beta(x)]=\left[T^{a}, T^{b}\right] \alpha_{a}(x) \beta_{b}(x)
$$

is again a gauge transformation. In the $\mathrm{NC}$ case, a natural generalization of the gauge transformations is $\delta_{\alpha} \phi=\alpha \star \phi$. The commutator of two consequent transformations reads

$$
\begin{aligned}
& \alpha(x) \star \beta(x)-\beta(x) \star \alpha(x)= \\
& =\frac{1}{2}\left[T^{a}, T^{b}\right]\left(\alpha_{a} \star \beta_{b}+\beta_{b} \star \alpha_{a}\right)+\frac{1}{2}\left\{T^{a}, T^{b}\right\}\left(\alpha_{a} \star \beta_{b}-\beta_{b} \star \alpha_{a}\right)
\end{aligned}
$$

This has to be a gauge transformation again. Therefore, the set of generators $T^{a}$ must be closed with respect to both commutators and anticommutators. This requirement imposes very severe restrictions on allowed gauge groups and their representations [12], which are not compatible with symmetries of the real World. In short, only the $U(n)$ type gauge generators in fundamental or adjoint representation are allowed ${ }^{2}$, though the standard model of elementary particles requires more.

2.1. The Seiberg-Witten map. In 1999 Seiberg and Witten made an amazing discovery [34]. They proposed a map which relates commutative and noncommutative gauge theories. Due to this map, gauge symmetries of $\mathrm{NC}$ models may be realized through standard commutative transformations of commutative fields. In this way arbitrary gauge group can be realized. Although the Seiberg-Witten map remains the main tool to study experimental consequences of noncommutativity through modifications of the standard model (see, e.g., [8] and references therein), this cannot be considered as a complete solution of the problem. The noncommutative fields are expressed as power series in $\theta$ with growing powers of commutative fields and derivatives (and just a few terms are actually known explicitly). It is clear, that not all effects can be studied in the framework of a $\theta$-expansion. In quantum case, the models obtained through the Seiberg-Witten map have (rather predictable) problems with renormalization, see [44].

\footnotetext{
${ }^{2}$ The use of semi-infinite Wilson lines allows to soften the restrictions on representations [1].
} 
2.2. Symplectic diffeomorphisms. Since it does not look possible to make an NC theory invariant with respect to all diffeomorphisms, it seems natural to consider a subalgebra generated by the vector fields of the form

$$
\xi^{\mu}(x)=\theta^{\mu \nu} \partial_{\nu} f(x),
$$

which preserves $\theta^{\mu \nu}$ (under standard action of diffeomorphisms on a tensor) and try to construct a gravity theory basing on such a symmetry [10]. The transformations generated by the fields (4) preserve the volume element. Therefore, one deals with the so called unimodular gravity theories. Although this approach gave rise to many interesting results over the recent years, the group of symplectic diffeomerphisms is too small to be the only symmetry of general relativity, even on an NC manifold.

2.3. Stability of NC Jackiw-Teitelboim model. Another idea how to achieve a richer symmetry structure in NC theories was suggested by twodimensional dilaton gravity models [23]. After a suitable field redefinition almost all interesting models of that type can be written in the form

$$
S_{\text {dil }}=\int d^{2} x \varepsilon^{\mu \nu}\left(\phi \partial_{\mu} \omega_{\nu}+\phi_{a} D_{\mu} e_{\mu}^{a}-\varepsilon^{a b} e_{\mu}^{a} e_{\nu}^{b} V(\phi)\right)
$$

where both kind of indices $a, b$ and $\mu, \nu$ take values $0,1, e_{\mu}^{a}$ is the zweibein, $\varepsilon^{\mu \nu}$ is an antisymmetric Levi-Civita symbol $\left(\varepsilon^{10}=\varepsilon_{01}=1\right) . \quad D_{\mu}$ is a covariant derivative with the spin-connection $\omega_{\mu} \varepsilon_{b}^{a}$. $\phi$ is a scalar field called the dilaton, and $\phi_{a}$ is an auxiliary field (which essentially generates the torsion constraint). Any choice of the potential $V(\phi)$ leads to a consistent model.

In the particular case of linear $V(\phi)$ one obtains the Jackiw-Teitelboim model $[25,36,6]$. This model is equivalent to a topological $S U(1,1)$ theory. The gauge group $S U(1,1)$ cannot be closed in the NC case, but $U(1,1)$ can. By extending the model to an $\mathrm{NC} U(1,1)$ topological theory one arrives at the action [9]

$$
S^{(0)}=\frac{1}{4} \int d^{2} x \varepsilon^{\mu \nu}\left[\phi_{a b} \star\left(R_{\mu \nu}^{a b}-2 \Lambda e_{\mu}^{a} \star e_{\nu}^{b}\right)-2 \phi_{a} \star T_{\mu \nu}^{a}\right],
$$

where $R_{\mu \nu}^{a b}$ and $T_{\mu \nu}^{a}$ are noncommutative generalizations of curvature and torsion which now depend on two connections, $\omega_{\mu}$ and $b_{\mu}$. There is also a new dilaton field $\psi$ which enters the action (6) through the combination $\phi_{a b}:=\phi \varepsilon_{a b}-i \eta_{a b} \psi$ with $\eta_{a b}=\operatorname{diag}(+1,-1)_{a b}$. Together with extending the gauge group one has to introduce new fields in the theory, but this is a relatively moderate price to pay since these new fields decouple in the commutative limit ${ }^{3}$ and, hence, can be made invisible for present day

\footnotetext{
${ }^{3} \mathrm{~A}$ similar procedure can be also done in higher dimensions (see, e.g, [16]), but there one has to add more fields, ant their decoupling in the commutative limit is not automatic.
} 
experiments. Besides, this model is surprisingly easy to quantize [38]. One can find all quantum corrections by the methods developed earlier in the commutative case [27].

It seems natural to look for a deformation of the action (6) which, in the analogy with the commutative action (5), would have a proper number of local symmetries, though, maybe, with a non-linear algebra, but still closed under the commutation and with the standard Leibniz rule. The action (6) is linear in the dilaton fields. To analyze the deformations one adds to (6) all possible quadratic terms without explicit derivatives on the dilatons which are also real and preserve global Lorentz symmetry ${ }^{4}$. For example, one can add $\varepsilon^{\mu \nu} \varepsilon_{a b} e_{\mu}^{a} \star e_{\nu}^{b} \star \phi^{2}$ with an arbitrary coefficient. There are seven independent terms [41]. In principle, one should also consider arbitrary deformations of local symmetries and the solve the conditions that the action is invariant under such symmetries. Fortunately, there is a short cut. One can use the canonical formalism [39] to check the closure of the constraint algebra. The result is negative: no quadratic deformation of (6) preserves the number of local symmetries.

In two dimensions there is a similar result on the $\kappa$-Poincare algebra which is quantum deformation of usual Poincare [29]. Gravity theories in two dimensions with local $\kappa$-Poincare symmetry but undeformed Leibniz rule are equivalent to undeformed theories with local Poincare symmetry [23].

\section{Twisted symmetries}

Since neither of the attempts to extend the standard (Lie algebra) approach to symmetries to $\mathrm{NC}$ theories was completely successful, one arrives at an idea to make a more substantial modification of the very concept of symmetries.

If we know the action of a symmetry generator $\alpha$ on the fields $\phi_{1}$ and $\phi_{2}$ then the action of $\alpha$ on a tensor product is defined by the so-called co-product $\Delta(\alpha)$. In the case of a Lie algebra symmetry the coproduct is primitive, $\Delta(\alpha)=\Delta_{0}(\alpha)=\alpha \otimes 1+1 \otimes \alpha$, so that we have the usual Leibniz rule

$$
\alpha\left(\phi_{1} \otimes \phi_{2}\right)=\Delta_{0}(\alpha)\left(\phi_{1} \otimes \phi_{2}\right)=\left(\alpha \phi_{1}\right) \otimes \phi_{2}+\phi_{1} \otimes\left(\alpha \phi_{2}\right) .
$$

The primitive coproduct $\Delta_{0}$ is not the only possible coproduct. To be able to discuss various coproducts systematically we need to make a Hopf algebra out of our symmetry generators. Consider a Lie algebra $G$ and its universal enveloping algebra $H=\mathcal{U}(G)$. Then $H$ is an associative unital

${ }^{4}$ In two dimensions $\theta^{\mu \nu} \simeq \varepsilon^{\mu \nu}$ is invariant under global Lorentz transformations. 
algebra. A coproduct is an algebra homomorphism $\Delta: H \rightarrow H \otimes H$ which satisfies the coassociativity relation

$$
(\Delta \otimes 1) \circ \Delta=(1 \otimes \Delta) \circ \Delta .
$$

To complete the Hopf algebra structure one also has to introduce an antipode and a counit, both satisfying certain relations with the coproduct and between themselves. We shall not need these elements. The interested reader can consult any textbook on Hopf algebras or on quantum groups. A nice simple introduction can be found in [35].

Suppose we have a twist element $\mathcal{F} \in H \otimes H$ satisfying the relation [32]

$$
(\mathcal{F} \otimes 1)(\Delta \otimes 1) \mathcal{F}=(1 \otimes \mathcal{F})(1 \otimes \Delta) \mathcal{F}
$$

(and another relation involving counit). Then we may define another (twisted) coproduct

$$
\Delta_{\mathcal{F}}=\mathcal{F} \Delta \mathcal{F}^{-1}
$$

(also twisting the counit and the antipode). Suppose now that our algebra $G$ contains space-time translations which are represented by partial derivatives. Then the twist element defined in eq. (2) above belongs to $H \otimes H$. One can check that the equation (10) is satisfied by (2) for $\Delta=\Delta_{0}$.

The action of a generator $\alpha$ on the star-product of fields is defined as follows

$$
\alpha\left(\phi_{1} \star \phi_{2}\right)=\mu_{\star}\left(\Delta_{\mathcal{F}}(\alpha) \phi_{1} \otimes \phi_{2}\right)=\mu \circ \mathcal{F}^{-1}\left(\Delta_{\mathcal{F}}(\alpha) \phi_{1} \otimes \phi_{2}\right)
$$

Here and everywhere below $\Delta_{\mathcal{F}} \equiv\left(\Delta_{0}\right)_{\mathcal{F}}=\mathcal{F} \Delta_{0} \mathcal{F}^{-1}$. In a sense, twisting pushes the generator $\alpha$ through the star-product, so that the star-product itself is not transformed. Therefore, it becomes much easier to construct invariants.

The idea to twist physical symmetries appeared already in [26], though with a different twist. The same twist as above but without analysing invariants was suggested first in [31]. The real break through came later, when twisted Poincare symmetry of noncommutative field theories was constructed [11, 42, 13]. Afterwards twisted conformal symmetries [30, 28], twisted diffeomorphisms $[2,3]$, and twisted gauge symmetries $[40,4]$ were constructed (to mention bosonic symmetries only). Let us consider in some detail twisted gauge symmetries [40,4]. This particular case is chosen since (i) it is rather simple, (ii) the topic is still quite controversial, and (iii) I have something new to say on this subject.

To be concrete, let us consider a theory describing some scalar field $\phi$ and gauge fields (connections) $A_{\mu}$. Gauge transformations of these fields with the parameter $\alpha(x)$ can be written as

$$
\alpha: \Phi \rightarrow \Phi+\delta_{\alpha} \Phi, \quad \delta_{\alpha} \Phi=R(\alpha) \Phi
$$


where

$$
\Phi \equiv\left(\begin{array}{c}
\phi \\
A_{\mu} \\
1
\end{array}\right), \quad R(\alpha)=\left(\begin{array}{ccc}
\alpha(x) & 0 & 0 \\
0 & \operatorname{ad}(\alpha) & -\partial_{\mu} \alpha \\
0 & 0 & 0
\end{array}\right) .
$$

The twisted coproduct reads in the $\theta$-expansion

$$
\begin{aligned}
\Delta_{\mathcal{F}}(\alpha)= & R(\alpha) \otimes 1+1 \otimes R(\alpha)-\frac{i}{2} \theta^{\mu \nu}\left(\left[\partial_{\mu}, R(\alpha) \otimes \partial_{\nu}+\partial_{\mu} \otimes\left[\partial_{\nu}, R(\alpha)\right]\right)\right. \\
& -\frac{1}{8} \theta^{\mu \nu} \theta^{\rho \lambda}\left(\left[\partial_{\mu},\left[\partial_{\rho}, R(\alpha)\right]\right] \otimes \partial_{\nu} \partial_{\lambda}+\partial_{\mu} \partial_{\rho} \otimes\left[\partial_{\nu},\left[\partial_{\lambda}, R(\alpha)\right]\right]\right. \\
& +\mathcal{O}\left(\theta^{3}\right) .
\end{aligned}
$$

Now one can start constructing invariants. One immediately finds a lot of invariants involving the scalar field and a very important invariant of the gauge field

$$
\operatorname{tr}\left(F_{\mu \nu} \star F^{\mu \nu}\right)
$$

constructed from an $\mathrm{NC}$ generalization of the field strength (bundle curvature)

$$
F_{\mu \nu}=\partial_{\mu} A_{\nu}-\partial_{\nu} A_{\mu}+A_{\mu} \star A_{\nu}-A_{\nu} \star A_{\mu} .
$$

The main advantage of this scheme is that any gauge group can be realized, but there are also drawbacks. One of them was noted already in [4] (for a more elaborate discussion see [21]). It was demonstrated that the action with the density (14) leads to inconsistent equations of motion unless one adds more vector fields with the values in the enveloping algebra of the original gauge algebra. However, this statement refers to just one possible deformation (14) of the commutative action for gauge fields. There are others. The key observation which has led to twisted gauge invariance of (14) is that $F_{\mu \nu}$ is twisted gauge covariant,

$$
\delta F_{\mu \nu}(x)=\alpha_{a}(x) \cdot\left[T^{a}, F_{\mu \nu}(x)\right] .
$$

The action of symmetry transformations in (16) is reducible. The components of $F_{\mu \nu}$ which belong to the Lie algebra transform through themselves. The same is true for the components which are proportional to anti-commutators $\left\{T^{a}, T^{b}\right\}$. Therefore, instead of one covariant object we have two:

$$
\begin{aligned}
& F_{\mu \nu}^{(1)}=\partial_{\mu} A_{\nu}-\partial_{\nu} A_{\mu}+\left(A_{a \mu} \star A_{b \nu}+A_{b \nu} \star A_{a \mu}\right) \frac{1}{2}\left[T^{a}, T^{b}\right], \\
& F_{\mu \nu}^{(2)}=\left(A_{a \mu} \star A_{b \nu}-A_{b \nu} \star A_{a \mu}\right) \frac{1}{2}\left\{T^{a}, T^{b}\right\},
\end{aligned}
$$


and instead of a single invariant (14) we have a two-parameter family

$$
\operatorname{tr}\left(F_{\mu \nu}^{(1)} \star F_{\mu \nu}^{(1)}+g_{1} F_{\mu \nu}^{(2)} \star F_{\mu \nu}^{(2)}+g_{2} F_{\mu \nu}^{(1)} \star F_{\mu \nu}^{(2)}\right) .
$$

It is quite possible (anyway, not excluded by the results of $[4,21]$ ) that for some choice of the parameters $g_{1}$ and $g_{2}$ and for some gauge groups the equations of motion become consistent.

Another criticism of the scheme based on twisted gauge transformations appeared in [14]. The authors of [14] claimed that this scheme contradicts the gauge principle since it implicitly assumes that the action of the Poincare generators appearing in the twist does not change representation of the gauge group. In other words, the twist operator is not covariant. According to a more moderate point of view [43] it is enough to have proper commutation relation between the symmetry generators and a coassociative coproduct, so that one has a Hopf algebra based on a proper Lie algebra. Gauge covariance of twist operator is not required. Regardless of whether covariance of the twist must or must not be included in a proper formulation of the gauge principle ${ }^{5}$ it is interesting on its own right to find a gauge covariant twist operator. Simply replacing partial derivatives by covariant derivatives does not work since the resulting star-product is non associative [15] (see also [24] for a related discussion in curved space).

The main ingredient of the construction below is a trivial connection ${ }^{6}$ $\tilde{A}_{\mu}=U\left(\partial_{\mu} U^{-1}\right)$, where $U(x)$ is an element of a finite-dimensional Lie group (which will later become the gauge group of some model). Obviously, the covariant derivatives $\tilde{\nabla}_{\mu}=\partial_{\mu}+\tilde{A}_{\mu}$ commute, and, as a consequence, the operator

$$
\mathcal{F}_{U}=\exp \mathcal{P}_{U}, \quad \mathcal{P}_{U}=-\frac{i}{2} \theta^{\mu \nu} \tilde{\nabla}_{\mu} \otimes \tilde{\nabla}_{\nu}
$$

satisfies the twist condition and can be used to construct an associative star product

$$
\phi_{1} \star_{U} \phi_{2}=\mu \circ \mathcal{F}_{U}^{-1}\left(\phi_{1} \otimes \phi_{2}\right) .
$$

More explicitly, if $\phi_{1}$ and $\phi_{2}$ transform according to representations $R_{1}$ and $R_{2}$ of the gauge group respectively,

$$
\phi_{1} \star_{U} \phi_{2}=\left(R_{1} \otimes R_{2}\right)(U) \cdot\left(R_{1}\left(U^{-1}\right) \phi_{1} \star R_{2}\left(U^{-1}\right) \phi_{2}\right),
$$

where $\star=\star_{1}$ is the Moyal product. The field $A_{\mu}$ does not belong to a linear representation of the gauge group. To construct a $\star_{U}$ product involving $A_{\mu}$

\footnotetext{
${ }^{5}$ This question may only be answered by studying physical consequences of both schemes.

${ }^{6}$ Trivial connections were used in star products already in [7] though without any relation to gauge symmetries.
} 
one has to exponentiate (13)

$$
\Phi \equiv\left(\begin{array}{c}
\phi_{1} \\
A_{\mu} \\
1
\end{array}\right), \quad R(U)=\left(\begin{array}{ccc}
R_{1}(U) & 0 & 0 \\
0 & \operatorname{Ad}(U) & U \partial_{\mu} U^{-1} \\
0 & 0 & 1
\end{array}\right) .
$$

The rest is straightforward, in particular we obtain

$$
A_{\mu} \star_{U} \phi_{1}=U\left(\left(U^{-1} \partial_{\mu} U+U^{-1} A_{\mu} U\right) \star\left(U^{-1} \phi_{1}\right)\right)+U \partial_{\mu} U^{-1} \cdot \phi_{1}
$$

(both $U$ and $A_{\mu}$ are taken in the representation $R_{1}$ ).

One can now proceed with constructing twisted gauge invariants in parallel to what we have outlined above in the case of usual Moyal product. In particular, one finds the property (16) for the field strength (15) with $\star$ replaced by $\star_{U}$. The new invariants constructed with $\star_{U}$ have a very important property which old invariants (constructed with $\star$ ) do not have: they become true gauge invariants with undeformed Leibniz rule if the transformation rules (12) for $\phi$ and $A_{\mu}$ are accompanied by a transformation of $U$, $\delta_{\alpha} U=\alpha \cdot U$. Therefore, we have just constructed an NC gauge theory with standard gauge transformations and an arbitrary gauge group. This makes twisting of gauge transformations unnecessary.

Now we have to return to the beginning of this article. Since $U$ is transformed under some symmetry transformations, it has to be a dynamical field. There are two distinct options.

(i) $U$ is an independent dynamical field. In this case one has to add a suitable action for $U$ to make corresponding equations of motion elliptic (or hyperbolic in the Minkowski space).

(ii) $U$ is a an already existing degree of freedom. One can, for example, identify $U$ with longitudinal degrees of freedom of $A_{\mu}$ by using the representation $A_{\mu}=U A_{\mu}^{T} U^{-1}+U \partial_{\mu} U$, where $A^{T}$ satisfies some gauge condition. E.g., the Lorenz condition $\partial^{\mu} A_{\mu}^{T}=0$ can be taken. Such a scheme depends, however, on gauge conditions imposed on $A_{\mu}^{T}$.

At present, it is not clear which of these two options (if any) is compatible with physics.

\section{Conclusions}

The main message of this paper is that usual concept of symmetries with the standard Leibniz rule seems to be insufficient for noncommutative field theories. The Leibniz rule (coproduct) must be deformed (twisted). Therefore, Hopf algebras arise in the field theory context. Over the last three years twisted counterparts were defined for almost all important physical symmetries. This subject is still a rather new one. Many problems remain 
open. We outlined possible solutions for two of such problems in the case of gauge symmetries.

We considered classical field theories only. An appropriate quantization scheme respecting the twisted symmetries is currently under debate, see recent papers $[37,45,20,5,33]$ and references therein.

Acknowledgement. I am grateful to A. Pinzul for interesting discussions. This work was supported in part by FAPESP.

\section{References}

[1] M. Arai, S. Saxell, A. Tureanu and N. Uekusa, "Circumventing the No-Go Theorem in Noncommutative Gauge Field Theory," arXiv:0710.3513 [hep-th].

[2] P. Aschieri, C. Blohmann, M. Dimitrijevic, F. Meyer, P. Schupp and J. Wess, "A gravity theory on noncommutative spaces," Class. Quant. Grav. 22 (2005) 3511-3532 [arXiv:hep-th/0504183].

[3] P. Aschieri, M. Dimitrijevic, F. Meyer and J. Wess, "Noncommutative geometry and gravity," Class. Quant. Grav. 23 (2006) 1883-1912 [arXiv:hep-th/0510059].

[4] P. Aschieri, M. Dimitrijevic, F. Meyer, S. Schraml and J. Wess, "Twisted gauge theories," Lett. Math. Phys. 78 (2006) 61-71 [arXiv:hep-th/0603024].

[5] A. P. Balachandran, A. Pinzul, B. A. Qureshi and S. Vaidya, "Twisted Gauge and Gravity Theories on the Groenewold-Moyal Plane," arXiv:0708.0069 [hep-th].

[6] B. M. Barbashov, V. V. Nesterenko and A. M. Chervyakov, "The solitons in some qeometrical field theories", Theor.Math.Phys. 40 (1979) 572 [Teor.Mat.Fiz. 40 (1979) 15].

[7] I. A. Batalin and I. V. Tyutin, "Quantum Geometry of Symbols and Operators," Nucl. Phys. B 345 (1990) 645-658.

[8] M. Buric, D. Latas, V. Radovanovic and J. Trampetic, "Nonzero Z $\rightarrow$ gamma gamma decays in the renormalizable gauge sector of the noncommutative standard model," Phys. Rev. D 75 (2007) 097701.

[9] S. Cacciatori, A. H. Chamseddine, D. Klemm, L. Martucci, W. A. Sabra and D. Zanon, "Noncommutative gravity in two dimensions," Class. Quant. Grav. 19 (2002) 4029 [arXiv:hep-th/0203038].

[10] X. Calmet and A. Kobakhidze, "Noncommutative general relativity," Phys. Rev. D 72 (2005) 045010 [arXiv:hep-th/0506157].

[11] M. Chaichian, P. P. Kulish, K. Nishijima and A. Tureanu, "On a Lorentz-invariant interpretation of noncommutative space-time and its implications on noncommutative QFT," Phys. Lett. B 604 (2004) 98-102 [arXiv:hep-th/0408069].

[12] M. Chaichian, P. Presnajder, M. M. Sheikh-Jabbari and A. Tureanu, "Noncommutative gauge field theories: A no-go theorem," Phys. Lett. B 526 (2002) 132 136 [arXiv:hep-th/0107037].

[13] M. Chaichian, P. Presnajder and A. Tureanu, "New concept of relativistic invariance in NC space-time: Twisted Poincare symmetry and its implications," Phys. Rev. Lett. 94 (2005) 151602 [arXiv:hep-th/0409096].

[14] M. Chaichian and A. Tureanu, "Twist symmetry and gauge invariance," Phys. Lett. B 637 (2006) 199-202 [arXiv:hep-th/0604025].

[15] M. Chaichian, A. Tureanu and G. Zet, "Twist as a Symmetry Principle and the Noncommutative Gauge Theory Formulation," Phys. Lett. B 651 (2007) 319-323 [arXiv:hep-th/0607179]. 
[16] A. H. Chamseddine, "Complexified gravity in noncommutative spaces," Commun. Math. Phys. 218 (2001) 283-292 [arXiv:hep-th/0005222].

[17] A. Connes, " $C^{*}$-algèbres et géométrie différentielle," C.R. Acad. Sci. Paris 290 (1980) 599-604.

[18] A. Connes, "Noncommutative geometry", Academic Press, 1994.

[19] M. R. Douglas and N. A. Nekrasov, "Noncommutative field theory," Rev. Mod. Phys. 73 (2001) 977 [arXiv:hep-th/0106048].

[20] G. Fiore and J. Wess, "On 'full' twisted Poincare' symmetry and QFT on MoyalWeyl spaces," Phys. Rev. D 75 (2007) 105022 [arXiv:hep-th/0701078].

[21] S. Giller, C. Gonera, P. Kosinski and P. Maslanka, "On the consistency of twisted gauge theory," Phys. Lett. B 655 (2007) 80-83 [arXiv:hep-th/0701014].

[22] D. Grumiller, W. Kummer and D. V. Vassilevich, "Dilaton gravity in two dimensions," Phys. Rept. 369 (2002) 327-430 [arXiv:hep-th/0204253].

[23] D. Grumiller, W. Kummer and D. V. Vassilevich, "A note on the triviality of kappa-deformations of gravity," Ukr. J. Phys. 48 (2003) 329-333 [arXiv:hepth/0301061].

[24] E. Harikumar and V. O. Rivelles, "Noncommutative gravity," Class. Quant. Grav. 23 (2006) 7551-7560 [arXiv:hep-th/0607115].

[25] R. Jackiw, in Quantum Theory Of Gravity, p. 403-420, S.Christensen (ed.) (Adam Hilger, Bristol, 1983).

[26] P. P. Kulish and A. I. Mudrov, "Twist related geometries on $q$-Minkowski space", Proc. Steklov Math. Inst. 226 (1999) 97-111 [arXiv:math.QA/9901019].

[27] W. Kummer, H. Liebl and D. V. Vassilevich, "Exact path integral quantization of generic 2-D dilaton gravity," Nucl. Phys. B 493 (1997) 491-502 [arXiv:grqc/9612012].

[28] F. Lizzi, S. Vaidya and P. Vitale, "Twisted conformal symmetry in noncommutative two-dimensional quantum field theory," Phys. Rev. D 73 (2006) 125020 [arXiv:hep-th/0601056].

[29] J. Lukierski, H. Ruegg, A. Nowicki and V. N. Tolstoi, "Q deformation of Poincare algebra," Phys. Lett. B 264 (1991) 331-338.

[30] P. Matlock, "Non-commutative geometry and twisted conformal symmetry," Phys. Rev. D 71 (2005) 126007 [arXiv:hep-th/0504084].

[31] R. Oeckl, "Untwisting noncommutative $\mathrm{R}^{* *} \mathrm{~d}$ and the equivalence of quantum field theories," Nucl. Phys. B 581 (2000) 559-574 [arXiv:hep-th/0003018].

[32] N. Reshetikhin, "Multiparameter quantum groups and twisted quasitriangular Hopf algebras," Lett. Math. Phys. 20 (1990) 331-335.

[33] M. Riccardi and R. J. Szabo, "Duality and Braiding in Twisted Quantum Field Theory," arXiv:0711.1525 [hep-th].

[34] N. Seiberg and E. Witten, "String theory and noncommutative geometry," JHEP 9909 (1999) 032 [arXiv:hep-th/9908142].

[35] R. J. Szabo, "Symmetry, gravity and noncommutativity," Class. Quant. Grav. 23 (2006) R199-R242 [arXiv:hep-th/0606233].

[36] C. Teitelboim, "Gravitation and Hamiltonian Structure in Two Space-Time Dimensions, Phys.Lett.B 126 (1983) 41;

[37] A. Tureanu, "Twist and spin-statistics relation in noncommutative quantum field theory," Phys. Lett. B 638 (2006) 296-301 [arXiv:hep-th/0603219].

[38] D. V. Vassilevich, "Quantum noncommutative gravity in two dimensions," Nucl. Phys. B 715 (2005) 695-712 [arXiv:hep-th/0406163].

[39] D. V. Vassilevich, "Constraints, gauge symmetries, and noncommutative gravity in two dimensions," Theor. Math. Phys. 148 (2006) 928-940 [Teor. Mat. Fiz. 148 (2006) 64-79] [arXiv:hep-th/0502120]. 
[40] D. V. Vassilevich, "Twist to close," Mod. Phys. Lett. A 21 (2006) 1279-1284 [arXiv:hep-th/0602185].

[41] D. V. Vassilevich, R. Fresneda and D. M. Gitman, "Stability of a noncommutative Jackiw-Teitelboim gravity," Eur. Phys. J. C 47 (2006) 235-240 [arXiv:hepth/0602095].

[42] J. Wess, "Deformed coordinate spaces: Derivatives," Lectures given at BW2003 Workshop on Mathematical, Theoretical and Phenomenological Challenges Beyond the Standard Model: Perspectives of Balkans Collaboration, Vrnjacka Banja, Serbia, 29 Aug - 2 Sep 2003. arXiv:hep-th/0408080.

[43] J. Wess, "Differential calculus and gauge transformations on a deformed space," Gen. Rel. Grav. 39 (2007) 1121-1134 [arXiv:hep-th/0607251].

[44] R. Wulkenhaar, "Non-renormalizability of Theta-expanded noncommutative QED," JHEP 0203 (2002) 024 [arXiv:hep-th/0112248].

[45] J. Zahn, "Remarks on twisted noncommutative quantum field theory," Phys. Rev. D 73 (2006) 105005 [arXiv:hep-th/0603231]. 